\section{Are You GS1-Compliant? One Hospital Pharmacy's Experience}

In 2004, the US Food and Drug Administration (FDA) issued a new rule making it a requirement for most prescription products to carry bar codes down to the dose level. However, the FDA did not enforce any standards, nor did it require manufacturers to include expiration dates or lot numbers. In 2010, the American Society of Health-System Pharmacists (ASHP) published a draft version of its statement on bar $\operatorname{codes}^{1}$ and put into place a centre that would accept reports of bar-coding problems. ${ }^{2}$ Health Canada has not yet adopted mandatory bar coding for drugs, but earlier this year, the Institute for Safe Medication Practices Canada and the Canadian Patient Safety Institute jointly endorsed the adoption of the GS1 global standard for automated identification of pharmaceutical products in Canada. ${ }^{3,4}$

In Canada, the pharmaceutical industry uses both Universal Product Code (UPC) and Global Trade Identification Number (GTIN) symbologies for bar-coded drug identification. The GTIN, which refers to a family of GS1 global data structures, employs 14 digits and can be encoded into various types of data carriers. The GTIN is compatible with existing standards such as the UPC and does not place any additional demands on scanning hardware. ${ }^{5}$ The GS1 DataBar symbology requires that the GTIN be preceded by an application identifier, for a total of 16 digits.

At the Centre hospitalier universitaire Sainte-Justine (CHUSJ), we have been using bar codes for many years, mainly for inventory management. Recently, we explored the concept of numeric identity for pharmacy products, whereby all relevant information for a given drug would be grouped within a single database (e.g., drug numbers, including applicable bar-code numbers; drug description; drug characteristics; drug images $)^{6}$ that can support the various software programs used throughout the medication-use process. We tested this "proof of concept" and implemented a web-based page to support our daily compounding production of nonsterile oral products with bar codes. ${ }^{7}$ Inspired by the Canadian Pharmaceutical Bar Coding Initiative, we revisited our bar-coding strategies.

First, we applied for advanced membership in GS1 Canada (at a cost of \$750). This allowed us to obtain our own 5-digit identifier for the CHUSJ Pharmacy Department. Any locally generated bar codes containing this identifier should be readable and interpretable both inside and outside our organization.

Second, we evaluated the feasibility of modifying our existing bar codes (e.g., 6 digits in code 39 symbology) to numbers of 16 digits or more, to comply with GS1 requirements. This would include redesign of our printed labels and the use of new printers for higher resolution.

Third, recognizing that the department produces not only drug doses, but also various types of drug information (e.g., drug labels, medication administration reports, preprinted orders), we decided that any information produced by the Pharmacy Department should also have a meaningful bar code.

Fourth, we explored the potential of automated identification. ${ }^{8}$ To attribute meaningful data encoded within a bar code, an automated identification prefix code can be applied. Aside from denoting the meaning, the prefix indicates the format of the data that follow. Automated identifiers are available for identification, traceability, dates, quantity, locations, and other variables. Although our initial objective was to use automated identifiers to capture expiration dates and lot numbers, we realized that it would be difficult to determine applicable expiration dates once labels had been printed. Therefore, we decided to use a serialization number matching our production number, since the serialization number refers to a complete set of data in the relevant database.

We completed our analysis by determining a GTIN toponymy with and without additional automated identifiers. For instance, 16-digit GTIN bar codes will be used for local unit-dose packs, and longer bar codes, with additional automated identifiers, will be used for printed labels or reports related to individual orders on medication administration records and nonsterile compounded doses.

Let's take an example of the potential benefits of such a system. An order is validated by a pharmacist, and a drug label with a bar code is printed from the pharmacy information system. The bar code contains a GTIN plus a production number. That production number refers to a specific patient and a specific drug service in the pharmacy information system. The printed label is taken to the sterile room where it is scanned and associated with the UPC/GTIN bar code printed on the commercial antibiotic vial used. When the syringe containing the compounded product is ready, the drug label is scanned again and an alert is displayed on the dashboard of the unit coordinator, indicating that the medication is ready to be picked up by a nurse at the pharmacy. It is known that the antibiotic syringe should be administered to the patient within $12 \mathrm{~h}$. However, nobody comes to the pharmacy to pick up the syringe within the expected timeframe. The syringe is retrieved much later, and the label is scanned before the drug is administered to the patient. The bar-code reader displays an alert, indicating that the compounded product in the syringe has expired and should be discarded.

For some, it may seem too early to begin reflecting on optimal practices for hospital pharmacy bar-coding, given that Canadian standards are still emerging. We believe it is time to evaluate and share our experiences with this technology.

\section{References}

1. Draft ASHP statement on bar code verification during inventory, preparation, and dispensing of medications. Bethesda (MD): American Society of Health-System Pharmacists; [cited 2010 Jan 1]. Available from: www.ashp.org/DocLibrary/BestPractices/DraftDocs/DraftBarcode DispensingJan10.pdf 
2. ASHP drug product bar-code problem reporting center. Bethesda (MD): American Society of Health-System Pharmacists; [cited 2010 Apr 4]. Available from: www.ashp.org/Import/MEMBERCENTER/Sections/ SectionofPharmacyInformaticsandTechnology/Resources/BarCode.aspx

3. Canadian Pharmaceutical Bar Coding Project: joint technical statement on pharmaceutical automated identification and product database requirements. Toronto (ON): Institute for Safe Medication Practices Canada; 2010 [cited 2010 Jun 29]. Available from: www. ismp-canada.org/barcoding/download/CanPharmBarcode_Joint TechnicalStatement.pdf

4. Canadian Pharmaceutical Bar Coding Project. Toronto (ON): Institute for Safe Medication Practices Canada; [cited 2010 Apr 4]. Available from: www.ismp $\neg$ canada.org/barcoding/index.htm

5. What is GTIN? Chicago (IL): Bar Code Graphics Inc; 2009 [cited 2010 May 6]. Available from: www.gtin.info/

6. Bussières JF, Lebel D, Voytenko S, Vaquer G. Développement d'un concept et d'un processus de gestion de l'identité numérique d'un produit en établissement de santé. Can J Hosp Pharm 2009;62(5):406-414.

7. Lebel D, Vaquer G, Forest JM, Bussières JF. Utilisation d'une banque de données photographiques consultées par code-barres pour assister la préparation de seringues orales. Pharm Hosp 2009;44(3):114-124.

8. GS1 application identifiers. Chigaco (IL): Bar Code Graphics Inc; 2010 [cited 2010 May 6]. Available from: www.databar-barcode.info/ application\%2Didentifiers/

Denis Lebel, BPharm, MSC, FCSHP

Assistant Director

Jean-François Bussières, BPharm, MSc, MBA, FCSHP

Director

Department of Pharmacy and Research Unit in Pharmacy Practice

Centre hospitalier universitaire Sainte-Justine

Montréal, Quebec

\section{Explaining the RE-LY Trial}

In questioning the reliability of the RE-LY trial results, Tsang and others ${ }^{1}$ unfortunately based their concerns on a misinterpretation of the data that were published in the original trial report. ${ }^{2}$

The RE-LY trial, which had a study population of 18000 patients, compared 2 doses of dabigatran etexilate with warfarin in the largest randomized controlled trial of antithrombotic therapy for stroke prevention ever performed. The results showed that, relative to warfarin, dabigatran $110 \mathrm{mg}$ twice daily was associated with a similar rate of stroke and a lower rate of life-threatening, intracranial, major, minor, and total bleeding, whereas dabigatran $150 \mathrm{mg}$ twice daily reduced stroke as well as life-threatening intracranial and total bleeding.

The very substantial efficacy and safety benefits of dabigatran over warfarin in the RE-LY trial were associated with a modest excess of myocardial infarction, equivalent to 2 additional myocardial infarctions for every 1000 patients treated. The most likely explanation for this finding is the proven efficacy of warfarin for preventing myocardial infarction. Irrespective of the mechanism, the increase in myocardial infarction was substantially outweighed by the benefits of dabigatran: the reduction in hemorrhagic stroke alone was equivalent to 2.6-2.8 fewer hemorrhagic strokes for every 1000 patients treated. Among patients treated with dabigatran $150 \mathrm{mg}$ bid, major gastrointestinal bleeding was also greater than with warfarin, but this was a subcategory of all major types of bleeding, which was not increased with dabigatran. Thus, contrary to the claim of
Tsang and others, ${ }^{1}$ the RE-LY trial data conclusively demonstrated a net clinical benefit of both doses of dabigatran relative to warfarin.

Tsang and others ${ }^{1}$ criticized the choice of patient population in the RE-LY trial, citing the inclusion of nearly 6000 patients with a CHADS2 score of 0 or 1 , the CHADS(2) being a risk stratification index. Treatment guidelines, however, recommend either warfarin or acetylsalicylic acid for patients with one risk factor for stroke. ${ }^{3}$ Although some patients in the RE-LY trial had a CHADS(2) score of 0 , all patients in the trial had at least one risk factor for stroke, and the results demonstrated a consistent benefit of dabigatran over warfarin in the 6000 patients with a CHADS(2) score of 0 or 1 and in the 12000 patients with a CHADS(2) score of 2 or above. ${ }^{2}$

Tsang and others ${ }^{1}$ challenged the definition of systemic embolism and asked whether patients were screened for systemic emboli. It seems, however, that they have confused systemic arterial embolism with venous thromboembolism. There are no validated methods to screen for systemic embolism, and the trial used a standard definition for symptomatic events that is widely used in randomized controlled trials.

Tsang and others ${ }^{1}$ also raised questions about creatinine monitoring, potential adverse outcomes related to the use of amiodarone and quinidine, and the statistical analysis plan for the study. A creatinine measurement was required for all patients to assess their eligibility to enter the trial. As reported in the original article, ${ }^{2}$ dabigatran had superior efficacy and safety over warfarin, despite lack of routine creatinine screening during the trial, a finding that calls into question the relevance of the concerns raised by the correspondents. Patients who received amiodarone, a drug that can increase blood concentrations of dabigatran by up to $50 \%$, experienced a consistent benefit of dabigatran. Quinidine is rarely used in clinical practice, and very few patients received this drug during the trial. Finally, a per-protocol analysis yielded similar results to those obtained with an intention-to-treat analysis.

References

1. Tsang MP, Tejani A, Kuo I. Are the results of the RE-LY trial reliable? [letter]. Can J Hosp Pharm 2010;63(2):155-156.

2. Connolly SJ, Ezekowitz MD, Yusuf S, Eikelboom JW, Oldgren J, Parekh A, et al.; RE-LY Steering Committee and Investigators. Dabigatran versus warfarin in patients with atrial fibrillation. $N$ Engl J Med 2009; 361(12):1139-1151.

3. Fuster V, Rydén LE, Cannom DS, Crijns HJ, Curtis AB, Ellenbogen KA, et al.; American College of Cardiology/American Heart Association Task Force on Practice Guidelines; European Society of Cardiology Committee for Practice Guidelines; European Heart Rhythm Association; Heart Rhythm Society. ACC/AHA/ESC 2006 Guidelines for the Management of Patients with Atrial Fibrillation: a report of the American College of Cardiology/American Heart Association Task Force on Practice Guidelines and the European Society of Cardiology Committee for Practice Guidelines (Writing Committee to Revise the 2001 Guidelines for the Management of Patients With Atrial Fibrillation): developed in collaboration with the European Heart Rhythm Association and the Heart Rhythm Society. Circulation 2006;114(7):e257-e354.

John W Eikelboom, MBBS

Medicine

Stuart J Connolly, MD

Cardiology

McMaster University

Hamilton, Ontario 